\title{
Capacidade para o trabalho e envelhecimento funcional: análise Sistêmica da Literatura utilizando o PROKNOW-C (Knowledge Development Process - Constructivist)
}

\author{
Work ability and functional aging: a Systemic Analysis of the \\ Literature using ProkNow-C (Knowledge Development Process - \\ Constructivist)
}

João Eduardo Linhares ${ }^{1}$ Sergio Luiz Ribas Pessa ${ }^{1}$ Sandro César Bortoluzzi ${ }^{1}$ Roger Poglia da Luz ${ }^{1}$
${ }^{1}$ Universidade Tecnológica Federal do Paraná. R. Via do Conhecimento Km 01, Fraron. 85503-390 Pato Branco PR Brasil. jelinhares@gmail.com

\begin{abstract}
Brazilian Institute of Geography and Statistics (IBGE) data indicate that the elderly population of Brazil numbering 11\% in 2003, will attain $33.7 \%$ by 2060 . This aging process can be diagnosed from the capability workers possess for their labor activities. ProKnow-C was used to conduct the review, carrying out bibliometric and systemic analysis of the literature on the subject. The results led to the compilation of a portfolio with 29 papers, highlighting the most relevant journals, authors and keywords. Systemic analysis provided important results: survey methodology was applied on 26 papers, 21 of which were cross-sectional studies; in all the papers the WAI was associated with sociodemographic data; in 10 studies it was found that the work ability decreased while age increased and the practice of physical exercises was positively associated with the WAI in 11 studies. The results led to the perception that the WAI is an instrument that makes it possible to diagnose the situation of the working life of employees, and, in the case of low rates, the presence of early functional aging is detected. The study highlighted the importance of assessing aspects of the worker's social life, since these factors influence the work ability positively or negatively. Key words Work Ability Index (WAI), Occupational health, Aging, ProKnow-C, Review
\end{abstract}

Resumo Dados do IBGE apontam que a população idosa do Brasil que era de 11\%, em 2013, passará a ser de 33,7\%, em 2060. Este processo de envelhecimento pode ser diagnosticado a partir da capacidade que o trabalhador dispõe para suas atividades laborais. Para realizar uma revisão de literatura sobre o tema, utilizou-se o PROK$N O W-C$, realizando a análise bibliométrica $e$ sistêmica da literatura. Os resultados levaram a constituição de um portfólio com 29 artigos, destacando-se os periódicos de maior relevância, autores e palavras-chaves. A análise sistêmica proporcionou importantes resultados: a metodologia survey foi aplicada em 26 artigos; 21 estudos são cortes transversais; constatou-se que em 10 estudos a capacidade para o trabalho diminui com o avanço da idade e a prática de exercícios físicos foi associada positivamente com o ICT em 11 estudos. Os resultados confirmam que o ICT é um instrumento que permite diagnosticar a situação da vida laboral dos trabalhadores, e, em caso de baixos indices, a presença de um envelhecimento funcional precoce. O estudo possibilitou ressaltar a importanncia de avaliar aspectos da vida social do trabalhador, uma vez que estes fatores influenciam positiva ou negativamente na capacidade para o trabalho.

Palavras-chave Índice de capacidade para o trabalho, Saúde do trabalhador, Envelhecimento, PROKNOW-C, Revisão 


\section{Introdução}

Dados da Organização Mundial da Saúde indicam que a população mundial acima de 60 anos vai quase dobrar, de $12 \%$ para $22 \%$, entre 2015 e 2050. A França teve cerca de 150 anos para se adaptar a uma mudança de $10 \%$ para $20 \%$ na proporção da população com mais de 60 anos. No entanto, países como o Brasil, China e Índia terão pouco mais de 20 anos para passar pelo mesmo processo de adaptação ${ }^{1,2}$.

Esta configuração se confirmou em estudos do Instituto Brasileiro de Estatística e Geografia ${ }^{3}$, que apontaram para uma tendência de incremento na faixa etária de 60 anos. Nos anos 2000, $8,2 \%$ da população brasileira estavam nesta faixa etária e, em 2013, este percentual subiu para $11 \%$. Já as projeções para 2017, 2024 e 2060 apontam, respectivamente, que $12,5 \%, 15,7 \%$ e $33,7 \%$ da população terão 60 anos ou mais de idade.

A preocupação com o envelhecimento da população possui tanto um viés de riscos, quanto de oportunidades. Os riscos se referem à capacidade que governos, empresas e a própria população tem de se adaptar a esta mudança. Por outro lado, acredita-se que haverá uma demanda considerável na oferta de produtos e serviços para esta população mais idosa.

Dentro do contexto de gestão de recursos humanos e materiais, as organizações podem voltar seu olhar para o processo de envelhecimento, promovendo a capacidade para o trabalho dos empregados. Atuar nesta frente significa possibilitar melhorias na saúde do trabalhador e, em consequência, na produtividade da organização $0^{4}$.

Estudos revelaram que com o passar dos anos, a capacidade para o trabalho vai reduzindo, havendo um desequilíbrio entre as habilidades do trabalhador e as exigências do trabalho. Assim, não raramente, a capacidade do indivíduo diminui em ritmo maior que a demanda, em função das exigências do trabalho, de seu estado de saúde e de suas capacidades físicas e mentais, acarretando em um envelhecimento funcional precoce. No Brasil, a preocupação com o envelhecimento funcional precoce deve começar aos 45 anos de idade ${ }^{5-7}$.

Pesquisadores do Instituto Finlandês de Saúde Ocupacional (Finnish Institute of Occupational Health - FIOH) realizaram um estudo pioneiro na década de 1980, que resultou no desenvolvimento de um questionário denominado Índice de Capacidade para o Trabalho (ICT). O ICT é um índice de avaliação subjetiva, que leva em conta a perspectiva do próprio trabalhador, a fim de determinar suas condições de trabalho atuais e futuras ${ }^{8-11}$.

Por meio desta ferramenta, é possível determinar o ICT do trabalhador, mensurando sua Capacidade para o Trabalho (CT) e avaliando como está ocorrendo o envelhecimento funcional na organização. Esta ferramenta já foi traduzida para 26 línguas, transformando-a em um método mundialmente aplicado ${ }^{12}$.

O framework do ICT é constituído por 7 construtos, avaliando: (i) CT atual e comparada com a melhor que considera já ter tido na vida, (0 a 10 pontos); (ii) CT em relação às exigências do mesmo, por meio de duas questões sobre a natureza do trabalho (físico, mental ou misto) ( 2 a 10 pontos); (iii) número atual de doenças autorreferidas e diagnosticadas por médico, obtido a partir de uma lista de 51 doenças ( 1 a 7 pontos); (iv) perda estimada de aptidão para o trabalho devido a doenças, (1 a 6 pontos); (v) faltas ao trabalho motivadas por lesões ou doenças ( 1 a 5 pontos); (vi) prognóstico próprio sobre a CT (1, 4 ou 7 pontos); (vii) recursos mentais, obtidos pela ponderação das respostas de três questões (1 a 4 pontos) $)^{8}$.

A soma destes construtos resulta no ICT. O ICT baixo (score de 7 a 27) pressupõe uma resposta de ação por meio da qual o objetivo é de restaurar a capacidade para o trabalho do indivíduo. Já o ICT moderado (score de 28 a 36), sugere ações no sentido de melhorar a capacidade para o trabalho. Para o ICT bom (score de 37 a 43), o objetivo deve ser apoiar o indivíduo. Por fim, para o ICT ótimo (score 44 a 49), os esforços devem ser voltados à manutenção da capacidade ao trabalho.

O conceito de Capacidade para o Trabalho (CT) foi definido pelo Instituto Finlandês de Saúde Ocupacional como "o quão bem está, ou estará, um trabalhador presentemente ou em um futuro próximo, e quão capaz ele pode executar seu trabalho em função das exigências, de seu estado de saúde e capacidades físicas e mentais" ${ }^{\text {". }}$.

A capacidade para o trabalho é influenciada por diversos fatores. Entre estes, pode-se citar aqueles relacionados ao ambiente de trabalho, saúde do trabalhador e aspectos da vida social que influenciam na capacidade do trabalhador. Para cada grupo profissional a ser estudado, diferentes fatores sociodemográficos podem estar presentes, caracterizando o ICT como um constructo complexo ${ }^{13}$.

Diversas pesquisas têm sido realizadas utilizando-se do ICT no Brasil, indicando a necessidade de ampliar os estudos com esta ferramen- 
ta junto a outras categorias de trabalhadores ${ }^{14}$, tais quais, trabalhadores com 50 anos ou mais ${ }^{15}$, agentes comunitários de saúde ${ }^{16}$, profissionais da saúde $^{5}$, motoristas de ônibus ${ }^{17}$ e trabalhadores de empresa do setor elétrico ${ }^{14}$.

Os dados revelados pelo ICT, podem proporcionar um diagnóstico de como está o corpo funcional da organização, sobretudo em relação à existência de uma população com envelhecimento funcional precoce, em razão da baixa capacidade para o trabalho. Com a proximidade da idade de aposentadoria, os trabalhadores tendem a ter índices menores, demonstrando menor interesse no trabalho e uma aposentadoria prematura é ainda mais provável ${ }^{18}$.

Frente a este contexto, é de fundamental importância buscar a construção de um referencial teórico que permita identificar os fatores associados na CT dos indivíduos, observando o contexto de pesquisa. Para tanto, buscou-se responder quais são os critérios relevantes em uma revisão de literatura que permitam avaliar, a partir de um portfólio de artigos científicos, a capacidade para o trabalho frente ao envelhecimento funcional, em servidores públicos municipais.

O presente artigo teve por objetivo analisar e evidenciar, a partir de uma visão construtivista, os aspectos de destaque em um portfólio bibliográfico sobre o tema capacidade para o trabalho e envelhecimento funcional. A partir deste objetivo, pretendeu-se avaliar, com base na literatura, quais as metodologias de pesquisa para realizar os estudos, quais os conceitos de capacidade para o trabalho, quais as ferramentas utilizadas para avaliar a capacidade para o trabalho, bem como os principais achados da literatura sobre o tema.

A presente pesquisa foi estruturada da seguinte maneira: introdução e referencial teórico, método de revisão de literatura, apresentação dos resultados, discussão e conclusões.

\section{Métodos}

\section{Método de revisão}

A fim de proporcionar um levantamento bibliográfico estruturado, capaz de abranger estudos relevantes e que abordem o tema de capacidade para o trabalho e envelhecimento funcional, foi utilizado como método de revisão de literatura o PROKNOW-C, Knowledge Development Process-Constructivist ${ }^{19}$. O método propõe que o pesquisador possa formar um portfólio bibliográfico, a partir da sua área de interesse, obser- vando as delimitações e restrições intrínsecas ${ }^{20}$, e que os artigos que compõe este portfólio possam ser dotados de reconhecimento científico e alinhamento ao tema da pesquisa. O PROKNOW-C é um método difundido no meio científico ${ }^{20-23}$, e é composto por três etapas principais: seleção do portfólio de artigos; análise bibliométrica; e análise sistêmica ${ }^{19}$.

Na primeira etapa, foi realizada a busca dos artigos em bases de dados concernentes ao tema de interesse, onde houve a seleção dos artigos alinhados ao tema de pesquisa, observando as especificidades da população que se pretendeu observar, qual seja, servidores públicos municipais. A segunda etapa, composta pela bibliometria, buscou identificar dentre os artigos do portfólio a sua relevância, considerando o número de citações, os periódicos e autores que publicam estudos relativos ao tema, e palavras-chave mais utilizadas nos trabalhos. Já a terceira etapa, constituiu-se de uma análise sistêmica, por meio da qual se fez uma análise do conteúdo dos artigos do portfólio, a partir de lentes que auxiliam na construção da revisão de literatura. Estas lentes são capazes de demonstrar as lacunas da literatura, e as ferramentas adotadas para atender aos anseios dos pesquisadores ${ }^{19}$.

\section{Seleção do portfólio}

O tema de pesquisa, capacidade para o trabalho e envelhecimento funcional, foi trabalhado sob a perspectiva da Engenharia de Produção. Para abranger o tema sob esta óptica, as bases de dados utilizadas nesta pesquisa foram Web of Science e Scopus. A base Web of Science possui publicações de diversas áreas da ciência, e dá origem ao Journal Citation Reports ${ }^{\circledR}$ (JCR), que lista o fator de impacto de revistas científicas ${ }^{21}$. Já a base Scopus, também possui vasto banco de dados com publicações das mais diversas áreas da ciência e utiliza do SCImago Journal Rank (SJR) ${ }^{24}$, para obter o fator de avaliação de determinado periódico.

Para realizar a busca nas bases de dados, foram definidos três eixos de pesquisa: (i) Capacidade e Ambiente de Trabalho; (ii) Envelhecimento; (iii) Saúde e Bem-estar do Trabalhador. Os idiomas pesquisados foram português e inglês. $\mathrm{Na}$ sequência, foram definidas palavras-chave para cada eixo de pesquisa. Para o primeiro eixo, Capacidade e Ambiente de Trabalho, as palavras utilizadas foram: work ability, work capacity, functional ability, work conditions, demands of work e work contents. Para o eixo Envelhecimen- 
to, foram utilizadas: aging, aging workers, age, aging workforce, human factors, ageing e aged. Para o terceiro eixo, Saúde e bem-estar do Trabalhador, as seguintes palavras: occupational health, worker health, job satisfaction, occupational safety, care of work health, e health status. Estas palavras-chave geraram 272 diferentes combinações, proporcionando abrangência e especificidade para a pesquisa.

As buscas foram realizadas entre outubro e novembro de 2015. O processo de busca resultou em 2.620 publicações, sendo 656 artigos na base Web of Science e 1.964 artigos na base Scopus.

Na sequência, foram escolhidos dois artigos aleatórios a fim de aferir a aderência dos resultados, verificando se os dois artigos abrangiam as palavras-chave inicialmente propostas. A aderência foi considerada adequada, não sendo preciso incluir novas palavras-chave, passando à próxima etapa do PROKNOW-C.

Foi utilizado o gerenciador bibliográfico EndNote, para gerenciar as publicações, por meio da exportação dos artigos a partir das bases e a consequente importação no software. A partir de então, foi possível verificar a existência de artigos duplicados, haja vista que as duas bases possuem periódicos em comum. Foram excluídos 241 artigos duplicados, restando 2.379 publicações.

Passou-se a leitura dos títulos dos 2.379 artigos, a fim de verificar se estavam ou não alinhados ao tema capacidade para o trabalho e envelhecimento funcional. Assim, outras 1.950 publicações foram consideradas não alinhadas, resultando em um banco de 429 artigos com títulos alinhados à pesquisa.

O método PROKNOW-C visa, sobretudo, possibilitar ao pesquisador reunir um portfólio com reconhecimento científico e relevância ao tema de interesse. Para mensurar o reconhecimento científico, o método propõe a utilização do Google Acadêmico (http://scholar.google. com.br/), pesquisando os títulos dos artigos e verificando a quantidade de citações no referido website. Os dados coletados nesta etapa se referem ao mês de novembro de 2015.

Realizadas as pesquisas no Google Acadêmico constatou-se que o artigo mais citado recebeu 542 citações. Foi estabelecida a linha de corte de 10 citações, que representam $95,71 \%$ das citações, equivalentes a 225 artigos. Outros 204 artigos receberam 9 ou menos citações, dos quais 77 artigos não tiveram nenhuma citação constatada.
Na sequência do método, é recomendada a leitura dos resumos dos 225 artigos, para então classificá-los como alinhados ou não ao tema. Nesta fase, restaram 98 artigos (repositório A). Destes, foram identificados 301 autores, que formam o banco de autores de referência.

Para formação do repositório B, foram considerados artigos com 9 ou menos citações (total de 204). Classificaram-se estes artigos em: 94 artigos com menos de dois anos de publicação (mais recentes); e 110 artigos com mais de dois anos de publicação.

Dos 94 artigos mais recentes, procedeu-se à leitura dos resumos. Artigos com menos de dois anos de publicação não possuem potencial de serem citados em grande escala, em razão do tempo entre a realização da pesquisa, e posterior aceitação para publicação. Portanto, para que um artigo com menos de 2 anos de publicação seja citado por outro artigo inédito, pode levar um tempo maior. Considerando este critério, $42 \mathrm{ar}$ tigos recentes foram incluídos no repositório B.

Dos outros 110 artigos, com mais de dois anos de publicação, foram identificados seus autores e verificado se os mesmos estavam presentes no banco de autores do repositório A. Assim, 16 artigos possuíam autores integrantes do banco de autores, e foram submetidos à leitura dos resumos. O repositório B, portanto, foi formado somando 42 artigos mais recentes, e outros 16 artigos com mais de dois anos de publicação, mas com autores no banco de autores, totalizando assim 58 artigos.

Somando os repositórios A e B obtivemos um total 156 artigos, denominado repositório C.

$\mathrm{Na}$ última etapa da seleção do portfólio de artigos, foi realizada a verificação dos artigos disponíveis na íntegra. Parte dos artigos é de acesso livre, os quais foram integrados para a leitura. Outra parte são artigos com acesso restrito. Foi utilizado então o portal de periódicos da Coordenação de Aperfeiçoamento de Pessoal de Nível Superior (Capes), do Ministério da Educação, que disponibiliza o acesso a mais de 37 mil periódicos às instituições participantes ${ }^{25}$. Dos 156 artigos, 113 publicações foram obtidas na íntegra. Após a leitura integral destas publicações, 84 artigos não atendiam as especificidades da pesquisa, enquanto 29 publicações foram consideradas para a formação do portfólio bibliográfico, conforme apresentado no Quadro 1.

A formação do portfólio bibliográfico observou as etapas descritas na Figura 1. 
Quadro 1. Portfólio Bibliográfico resultante do método PROKNOW-C.

\begin{tabular}{|c|c|}
\hline Título & Autor \\
\hline Ageing and fitness to work. & Chan et al. ${ }^{26}$ \\
\hline $\begin{array}{l}\text { An approach of ergonomics and management in occupational health for a society of } \\
\text { aging workers. }\end{array}$ & Kumashiro $\mathrm{M}^{27}$ \\
\hline $\begin{array}{l}\text { Capacidade para o trabalho de trabalhadores de empresa de tecnologia da } \\
\text { informação. }\end{array}$ & Monteiro e Fernees $^{28}$ \\
\hline $\begin{array}{l}\text { Capacidade para o trabalho, sintomas osteomusculares e qualidade de vida } \\
\text { entre agentes comunitários de saúde em Uberaba, Minas Gerais. }\end{array}$ & Paula et al. ${ }^{16}$ \\
\hline $\begin{array}{l}\text { Changes in stress symptoms and their relationship to changes at work in 1981-1992 } \\
\text { among elderly workers in municipal occupations. }\end{array}$ & Huuhtanen ${ }^{29}$ \\
\hline Changes in the work ability of active employees over an 11-year period. & Ilmarinen et al. ${ }^{30}$ \\
\hline $\begin{array}{l}\text { Does perceived work ability improve after a multidisciplinary preventive program } \\
\text { in a population with no severe medical problems? the finnish public sector study. }\end{array}$ & Saltychev et al. ${ }^{31}$ \\
\hline $\begin{array}{l}\text { Effectiveness of a worksite exercise program with respect to perceived work ability } \\
\text { and sick leaves among women with physical work. }\end{array}$ & Nurminen et al. ${ }^{32}$ \\
\hline $\begin{array}{l}\text { Effects of ergonomic and health training on work interest, work ability and health } \\
\text { in elderly public urban transport drivers. }\end{array}$ & Karazman et al. ${ }^{18}$ \\
\hline Evaluating work ability and quality of life for clinical nurses in Taiwan. & Chiu et al. ${ }^{33}$ \\
\hline $\begin{array}{l}\text { Factors associated with work ability and perception of fatigue among nursing } \\
\text { personnel from Amazonia. }\end{array}$ & Vasconcelos et al. ${ }^{34}$ \\
\hline Health and work ability among office workers. & Martinez e Latorre ${ }^{2}$ \\
\hline Health Conditions and Factors Related to the Work Ability of Teachers. & Vedovato e Monteiro ${ }^{35}$ \\
\hline Identifying work ability promoting factors for home care aides and assistant nurses. & Larsson et al. ${ }^{36}$ \\
\hline Investigating the work ability of older employees. & Williams e Crumpton $^{37}$ \\
\hline $\begin{array}{l}\text { Mobbing, stress, and work ability index among physicians in Bosnia and } \\
\text { Herzegovina: Survey study. }\end{array}$ & Pranjic et al. ${ }^{38}$ \\
\hline $\begin{array}{l}\text { Organizational practices, work demands and the well-being of employees: a follow- } \\
\text { up study in the metal industry and retail trade. }\end{array}$ & Tuomi K et al. ${ }^{39}$ \\
\hline $\begin{array}{l}\text { Physical requirements associated with the work of aging workers in the European } \\
\text { Union. }\end{array}$ & Ilmarinen $^{40}$ \\
\hline $\begin{array}{l}\text { Predictors of lower work ability among emergency medicine employees: The } \\
\text { Croatian experience. }\end{array}$ & Klasan et al. ${ }^{41}$ \\
\hline Predictors of work ability in occupations with psychological stress. & Seibt et al. ${ }^{42}$ \\
\hline Promotion of work ability, the quality of work and retirement & Tuomi et al. ${ }^{4}$ \\
\hline $\begin{array}{l}\text { Satisfação no trabalho e capacidade para o trabalho entre docentes } \\
\text { universitários }\end{array}$ & $\begin{array}{l}\text { Marqueze e } \\
\text { Castro Moreno }^{43}\end{array}$ \\
\hline Teachers' voice disorders and loss of work ability: A case-control study & Giannini et al. ${ }^{44}$ \\
\hline Test-retest reliability of the Work Ability Index questionnaire & De Zwart ${ }^{45}$ \\
\hline $\begin{array}{l}\text { The relation of age, work ability index and stress-inducing factors among bus } \\
\text { drivers. }\end{array}$ & Kloimuller et al. ${ }^{46}$ \\
\hline The work ability index and functional capacity among older workers. & Padula et al. ${ }^{15}$ \\
\hline $\begin{array}{l}\text { Validity and reliability of the Brazilian version of the Work Ability Index } \\
\text { questionnaire. }\end{array}$ & Martinez et al. ${ }^{14}$ \\
\hline $\begin{array}{l}\text { Work ability assessment in a worker population: comparison and determinants of } \\
\text { Work Ability Index and Work Ability score }\end{array}$ & El Fassi et al. ${ }^{47}$ \\
\hline Work ability of health care shift workers: What matters? & Fischer et al. ${ }^{48}$ \\
\hline
\end{tabular}

Fonte: dados da pesquisa (2015). 


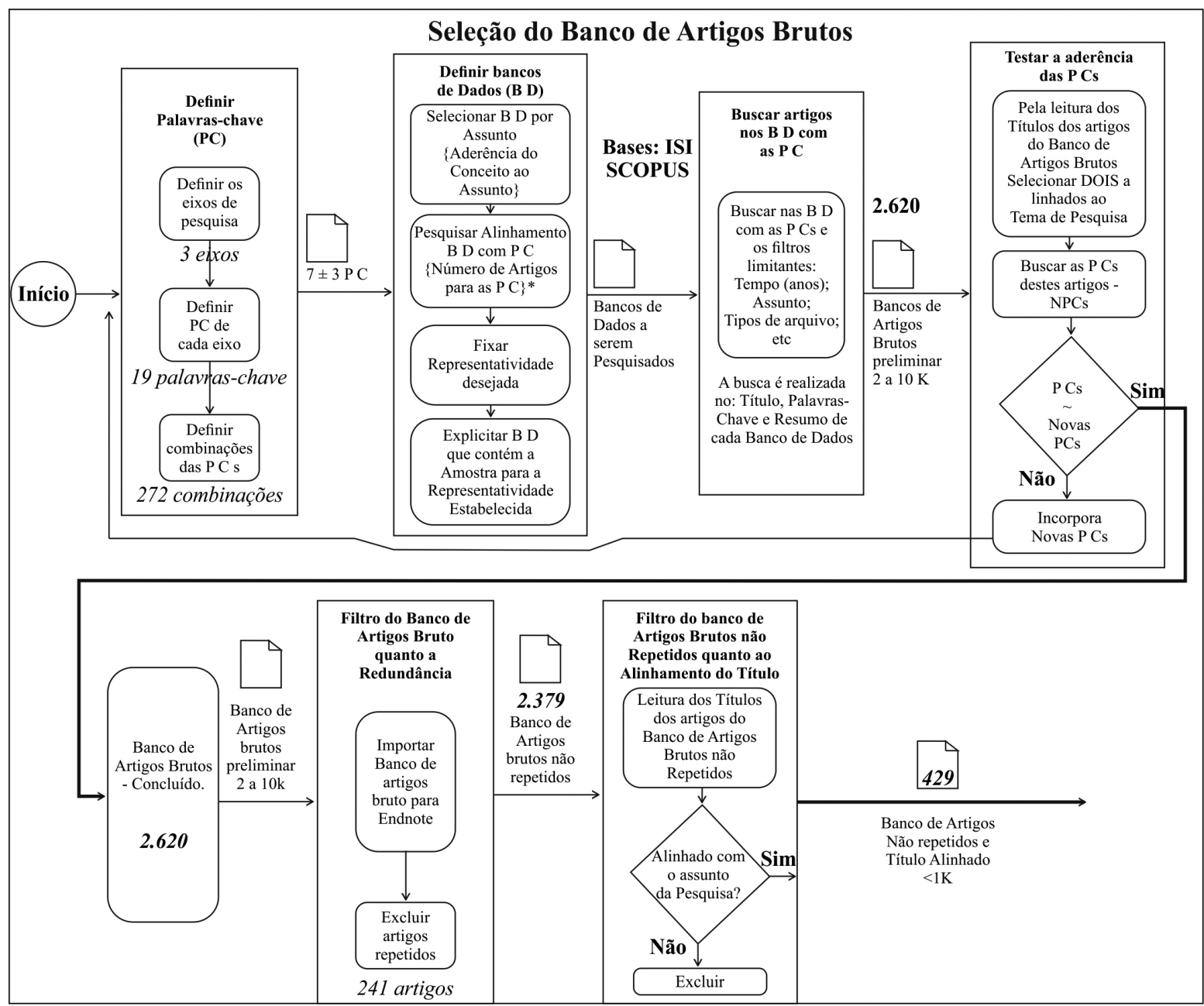

Figura 1. Fluxograma de seleção do portfolio bibliográfico.

continua

\section{Resultados}

\section{Análise bibliométrica}

Na proposta do $P R O K N O W-C$, a primeira avaliação bibliométrica a ser feita leva em consideração os periódicos do portfólio bibliográfico e de suas referências. A busca revelou que o periódico de maior destaque foi Scandinavian Journal of Work Environment \& Health com 4 artigos no portfólio e outros 42 artigos nas referências do portfólio. Já a "Revista de Saúde Pública", com 2 publicações no portfólio e outras 20 nas referências, é o periódico de destaque dentre as referências. Em meio ao portfólio bibliográfico, ainda se destacam Occupational and Environmental Medicine, com 4 artigos no portfólio e 14 nas referências, e International Journal of Industrial Ergonomics, com 3 artigos no portfólio e 2 nas referências. É importante destacar que foi no periódico "Scandinavian Journal of Work Environment \& Health", que aconteceram as primeiras publicações dos estudos do Instituto Finlandês de Saúde Ocupacional $^{29,30}$. Também é possível verificar que os periódicos "Revista de Saúde Pública", "Cadernos de Saúde Pública" e "Ciência \& Saúde Coletiva", são os periódicos brasileiros que mais se destacaram nesta pesquisa, dando origem a publicações importantes na área de capacidade para o trabalho e envelhecimento funcional no país (Gráfico 1).

A partir de pesquisas realizadas no site Google Acadêmico, foi verificado o reconhecimento científico dos artigos. Dos 29 artigos do portfólio, 3 apresentaram maior destaque: Ilmarinen et al. ${ }^{30}$ com 334 citações; Tuomi et al. ${ }^{4}$, com 303 citações; e De Zwart ${ }^{45}$ com 214 citações. 


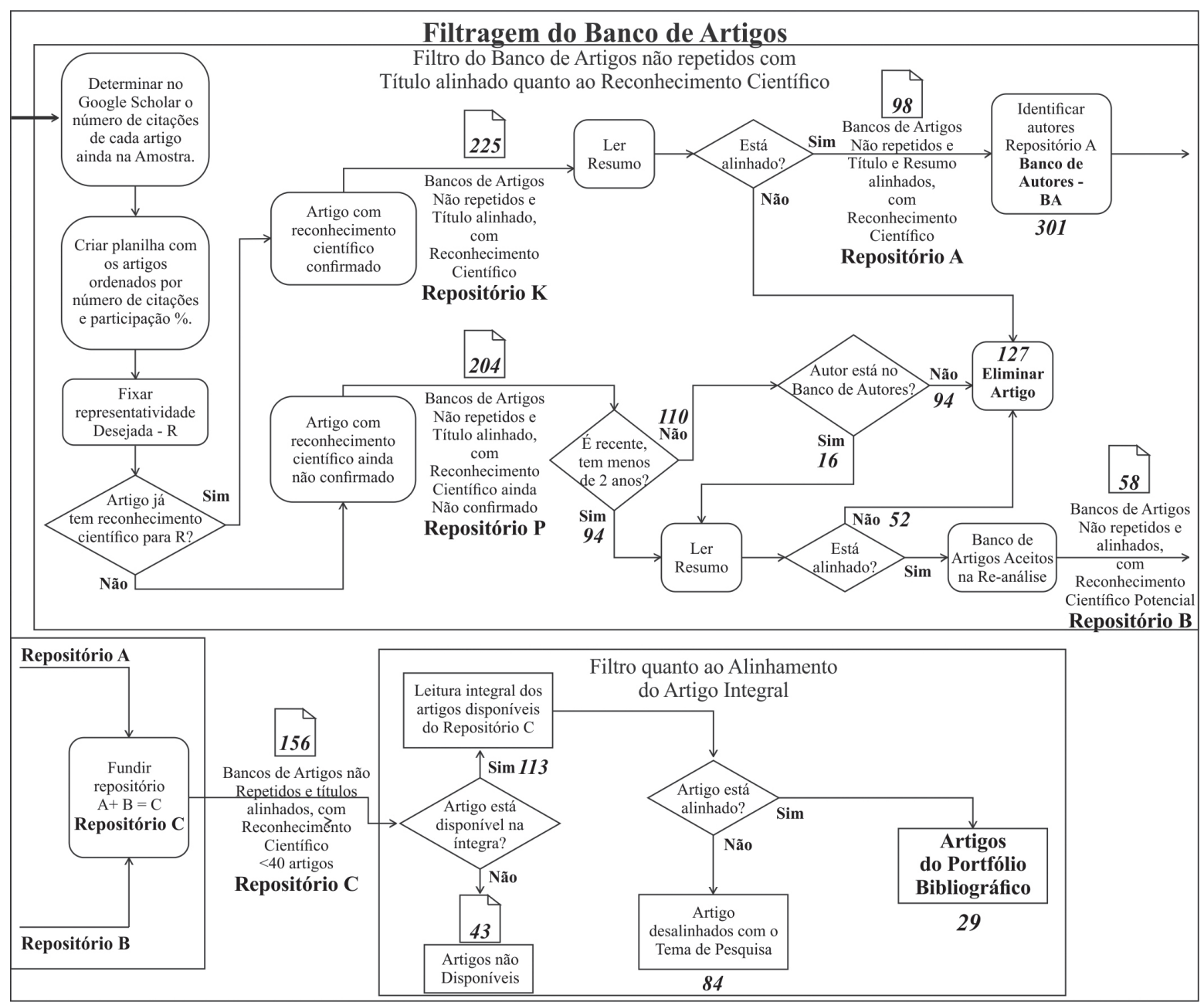

Figura 1. Fluxograma de seleção do portfolio bibliográfico.

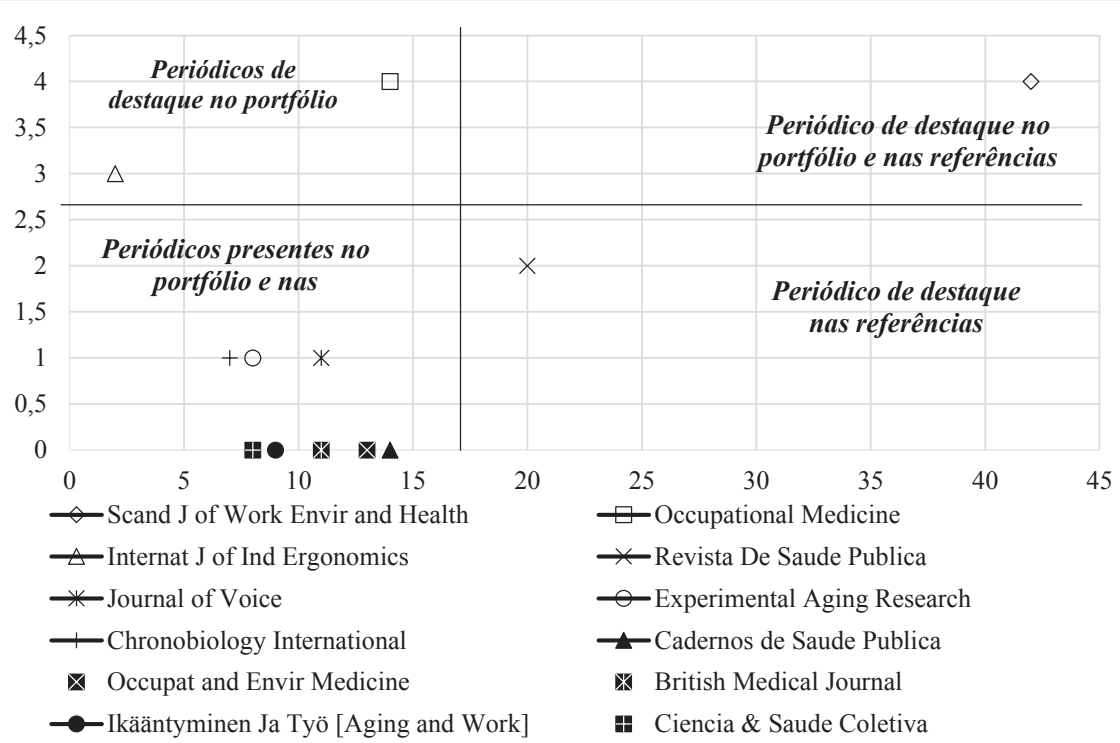

Gráfico 1. Número de publicações das referências do Portfólio Bibliográfico por periódico.

Fonte: dados da pesquisa 2015. 
Os autores de maior destaque no portfólio foram Juhani Ilmarinen, com 5 artigos; Frida Marina Fischer, com 4; Maria do Rosário Dias de Oliveira Latorre, com 4 artigos. Dentre as referências, novamente Juhani Ilmarinen, desta vez com 26 artigos é o autor de maior destaque; seguido por Maria do Rosário Dias de Oliveira Latorre, com 14 artigos; Kaija Tuomi, com 10 publicações; Frida Marina Fischer, com 9 artigos; e Clas-Håkan Nygård, também com 9 artigos. Dos autores de destaque, encontra-se 3 pesquisadores finlandeses e 2 brasileiros, revelando a importância do trabalho destes estudiosos para o desenvolvimento do tema (Gráfico 2).

$\mathrm{Na}$ análise das palavras-chave constantes do portfólio, temos a presença das seguintes palavras: "health" em 7 artigos; "public, environmental \& occupational health" também em 7; "work ability" presente em 6 artigos; e "job satisfaction" constante de 5 artigos. As palavras-chave "aging workers", "employees" e "occupational health" apareceram 4 vezes.

\section{Análise sistêmica}

A terceira etapa do PROKNOW-C buscou avaliar o conteúdo dos artigos do portfólio bibliográfico, por meio de lentes metodológicas. Uma lente, ou perspectiva teórica, visa delimitar e orientar o pesquisador na realização de sua pesquisa, buscando moldar, por exemplo, os tipos de questão ou a forma de coleta de dados ${ }^{49}$. Portanto, as lentes padronizam a busca do pesquisador, a fim de que possa observar em todo o portfólio os mesmos aspectos e, desta maneira, fazer um estudo comparativo relevante.

O ProKnow-C foi desenvolvido diante da ausência de um método de revisão bibliográfica na área de estudos de Avaliação de Desempenho ${ }^{50}$. Portanto, as lentes metodológicas aplicadas neste estudo foram adaptadas a partir das pesquisas de Avaliação de Desempenho ${ }^{21,50,51}$.

Como primeira lente aplicada, buscou-se descobrir a metodologia aplicada nos artigos, bem como a abordagem e a delimitação temporal adotada. Quanto à metodologia, houve 1 estudo de caso controle ${ }^{44}, 2$ artigos de revisão de literatu$\mathrm{ra}^{26,27}$ e 26 estudos tipo survey. Quanto à abordagem, os estudos de revisão de literatura não estão inclusos na contagem. Verificou-se que 6 estudos são longitudinais ${ }^{18,29-32,47}$; e 21 estudos são cortes transversais.

Destaca-se que o uso da metodologia survey na maioria dos estudos pode ser associado ao fato de que o ICT é instrumento do tipo questionário autoaplicável. Este tipo de instrumento permite a coleta de dados em grandes escalas, possibilitando a análise de uma amostra significativa do problema ${ }^{52}$, como é característico de estudos tipo survey.

A abordagem temporal dos estudos indicou uma presença maior de estudos de corte transversal (77\%), ante aos longitudinais (33\%). Estudos longitudinais exigem maior esforço por parte dos pesquisadores, pois a produção de resultados implica na análise de dados em série de vários

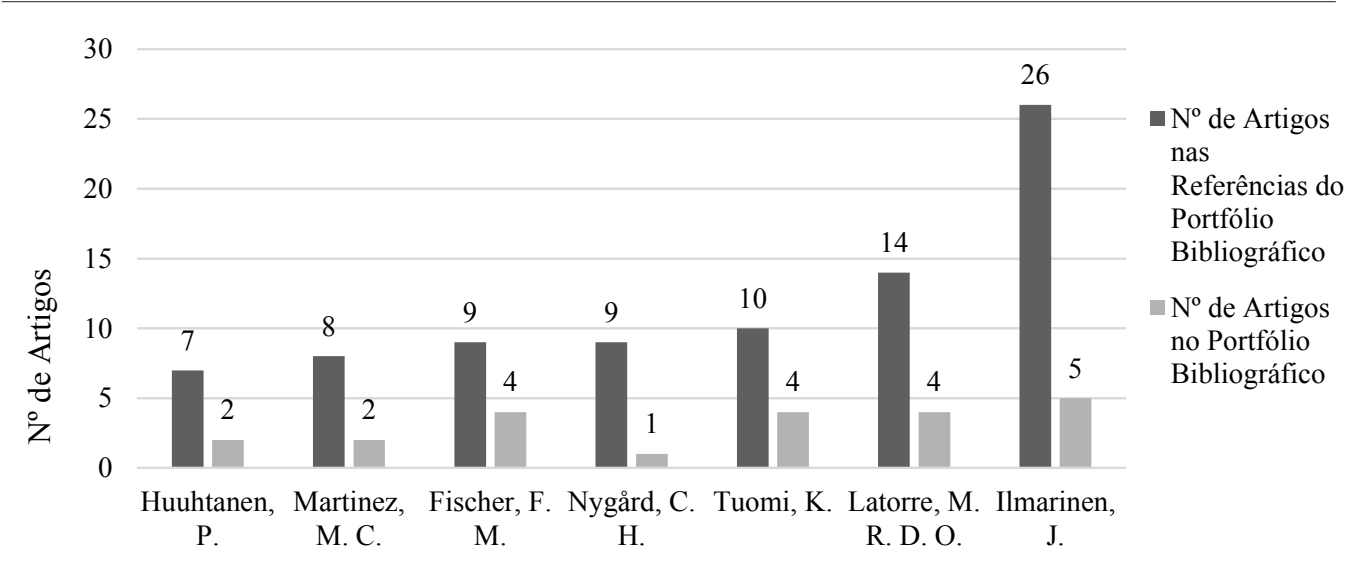

Autores do Portfólio Bibliográfico

Gráfico 2. Número de publicações por autor, no portfólio e nas referências do portfólio bibliográfico. 
anos. Contudo, os resultados apresentam maior consistência, e seu reconhecimento científico tende a ser maior.

Por outro lado, os estudos transversais contribuem para um diagnóstico mais imediato da situação, podendo constatar a existência de um envelhecimento funcional precoce, derivado da baixa capacidade para o trabalho dos empregados.

Na segunda lente aplicada nesta análise sistêmica, verificou-se a forma como os dados foram coletados, as categorias profissionais envolvidas nos estudos, o número de intervenções e o país ou região de abrangência.

A coleta de dados se deu de forma semelhante em 25 artigos, ou seja, por meio da aplicação de questionários, característica principal da metodologia survey. Nos 2 estudos de revisão de literatura, a coleta de dados foi realizada com aspectos subjetivos e abordagem qualitativa ${ }^{26,27}$. Outros dois estudos tiveram coleta de dados diferenciadas. No primeiro deles, um estudo envolvendo mulheres, além da aplicação de questionários foram realizados ensaios clínicos aleatórios ${ }^{32}$. Já no estudo que envolveu distúrbios de voz com professores, a coleta de dados incluiu avaliações vocais e laringoscopias ${ }^{44}$. Isto demonstra que, dependendo do problema de pesquisa, faz-se necessário realizar um diagnóstico mais aprofundado, com a utilização de ferramentas específicas ou complementares.

O número de intervenções e os profissionais estudados variaram de acordo com os objetivos de cada pesquisa. Dos 27 estudos - excluindo os estudos de revisão de literatura - em 2 casos envolveram trabalhadores administrativos ${ }^{6,28} ; 2$ trabalhadores de indústrias ${ }^{39,45}$; outros 2 com motoristas de ônibus ${ }^{18,46}$; já 4 estudos foram realizados com profissionais da educação $0^{35,42-44} ; 8$ com trabalhadores da área da saúde ${ }^{16,31,33,34,36,38,41,48}$; e 9 com trabalhadores de diversas áreas ${ }^{4,14,15,29,30,32,37,40,47}$ (Gráfico 3).

Os artigos do portfólio obtidos por meio do PROKNOW-C, respaldam a abrangência de estudos já realizados utilizando-se o ICT. Observou-se que as categorias profissionais envolvidas envolvem tanto trabalhos com demandas físicas quanto mentais, respaldando, portanto, que o ICT pode ser estendido às mais variadas categorias de trabalhadores, incluindo-se servidores públicos municipais.

Quanto ao país ou região de abrangência dos estudos, 10 foram realizados no Brasil $^{6,14,15,16,28,34,35,43,44,48}$, seis na Finlândia ${ }^{4,29,30-32,39}$, quatro na Alemanha ${ }^{18,42,45,46}$, e outras regiões com

\section{Quantidade de Estudos}
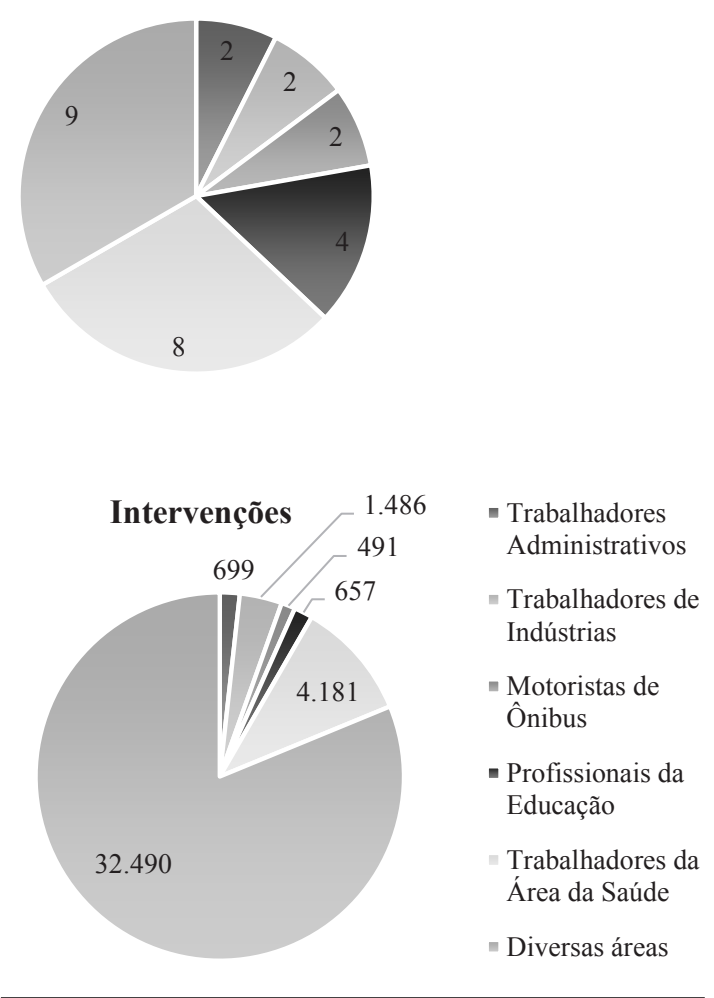

Gráfico 3. Número de estudos e intervenções em relação à categoria profissional objeto do estudo.

Fonte: dados da pesquisa 2015.

um estudo cada: Bósnia e Herzegovina ${ }^{38}$, Croá$\mathrm{cia}^{41}$, Estados Unidos da América ${ }^{37}$, Luxemburgo $^{47}$, Suécia ${ }^{36}$, Taiwan ${ }^{33}$ e União Europeia ${ }^{40}$. Estes dados corroboram a aplicabilidade do ICT em várias línguas ${ }^{12}$. Dentre o portfólio restaram 10 artigos com trabalhadores brasileiros, demonstrando a viabilidade da utilização do ICT no Brasil.

A terceira lente está voltada a identificar se os conceitos de capacidade para o trabalho dos artigos convergem com a afiliação teórica adotada, e quais ferramentas são utilizadas além do ICT. A afiliação teórica deste estudo parte dos conceitos e pesquisas desenvolvidas pelo Instituto Finlandês de Saúde Ocupacional, e do ICT ${ }^{8}$. Todos os 29 artigos do portfólio utilizam o ICT como ferramenta, e o conceito de capacidade para o trabalho converge com aquele desenvolvido pelos pesquisadores finlandeses. Este resultado demonstrou a eficácia do ProKnow-C em atender 
a especificidade da pesquisa, sobretudo no que se refere à busca de literatura focada na área da pesquisa.

Cada estudo possui objetivos próprios, o que implica na utilização de uma ou mais ferramentas para desenvolver as análises. Em 10 artigos verificou-se que o ICT foi aplicado comparando com dados sociodemográficos $\cos ^{4,26-28,35,37,39,40,45,47}$. Cinco estudos investigaram também aspectos de qualidade de vida (questionários SF-36 e WHOQOF-BREF $)^{6,14,16,33,41}$. Ainda, a abordagem do stress esteve presente em cinco publicações ${ }^{29,38,41,46,48}$.

A avaliação entre o ICT e problemas relacionados ao sistema osteomuscular foi abordada em três artigos, que utilizaram o Questionário Nórdico de Sintomas Osteomusculares (QNSO) ${ }^{16,32,36}$. A Avaliação Ergonômica do Trabalho (AET) esteve presente em duas publicações ${ }^{29,30}$. Também foram verificadas percepções de fadiga em dois estudos $^{34,48}$, e o nível de satisfação no trabalho em dois artigos ${ }^{6,43}$. Outras publicações ainda incluíram análises específicas como: avaliação de assédio moral e personalidade do indivíduo ${ }^{38}$; condições de produção vocal de professores ${ }^{44}$; demandas de trabalho ${ }^{36}$; percepção de capacidade para o trabalho ${ }^{31}$; questionário efeito tipologia (ETQ) ${ }^{18}$; testes físicos ${ }^{15}$; e índice de idade funcional ${ }^{42}$.

Esta lente apresenta as diversas nuances que o ICT pode apresentar, e sua vasta aplicação no campo de saúde ocupacional. São vários casos em que o ICT é associado a fenômenos relacionados à saúde do trabalhador, tal como estresse, fadiga, problemas osteomusculares. Em outros casos, o ICT é associado ao comportamento e a percepções da satisfação do trabalho, qualidade de vida e demandas de trabalho. Estas associações proporcionaram aprofundar os estudos realizados, buscando identificar as causas e propor adoção de medidas.

Outra avaliação feita sobre o conteúdo do portfólio foi quanto aos dados sociodemográficos investigados. Estes indicadores são fundamentais para associar os resultados do ICT com aspectos da vida social e laboral do indivíduo, caracterizando os grupos e instruindo as análises.

No portfólio constatou-se que há casos em que os indicadores sociodemográficos se restringiram a informações básicas como idade, gênero, estado civil e nível de escolaridade, o que aconteceu em 15 casos $^{4,14,18,27,29-32,36-38,40,43,45,46}$.

Os demais estudos incluíram questões específicas de dados sociodemográficos, com destaque para: oportunidades de adquirir novas informações e habilidades, participação e oportunidades de recreação e lazer ${ }^{28,33,42}$; tabagismo e obesida- $\mathrm{de}^{28,44,47}$; recursos financeiros ou responsabilidade sobre a renda familiar ${ }^{6,15,16,33,48}$; cuidar de crianças ou número de filhos ${ }^{41,48}$; trabalhos em turnos ${ }^{48}$; realização de tarefas domésticas ${ }^{28}$; e prática de exercícios físicos ${ }^{34,35,39}$.

Os artigos do portfólio demonstram que há diversas variáveis independentes a serem investigadas e associadas ao ICT, demonstrando que o indivíduo não está separado de sua vida social, e visando descobrir quais são os fatores que exercem maior influência sobre o índice.

Verificou-se que cuidar de crianças ou número de filhos ${ }^{41,48}$, é um fator que influencia negativamente na CT. Isto porque esta responsabilidade leva ao trabalhador a ter uma segunda jornada de trabalho, desta vez, em casa. No entanto, em nenhum dos artigos do portfólio, foi investigado se cuidar de pessoas idosas ou portadores de necessidades especiais pode ter efeito semelhante de uma sobrecarga na vida social, e, consequentemente, uma CT menor. Assim, destaca-se como uma lacuna constatada na literatura.

A última lente aplicada se refere aos resultados apresentados nos artigos do portfólio. É a partir destas decorrências que se pode distinguir a necessidade do uso de outras ferramentas para verificar a capacidade para o trabalho dos indivíduos.

É importante ressaltar que os fatores ambientais ou da vida social do trabalhador podem influenciar positiva ou negativamente na CT. Uma das variáveis mais analisadas nos estudos se refere à idade, e a maioria dos resultados aponta que com o passar dos anos a aptidão para o trabalho dos indivíduos diminui, conforme se abstraiu em 10 estudos $^{15,26,33,36,37,39,40,44,46,47}$.

Esta constatação vem de encontro ao que pesquisadores delinearam a respeito do uso do ICT como um instrumento de avaliação da existência do envelhecimento funcional precoce $e^{5,30}$. A compreensão de que há um declínio da habilidade do trabalhador com o passar dos anos, e que sua aptidão decai, não se configura como uma infringência do trabalhador ao sistema de trabalho, mas sim que é um processo decorrente do avanço cronológico e/ou perda do entusiasmo com consequente declínio da capacidade funcional. Portanto, não se pode afirmar que o indivíduo deva se enquadrar em um determinado padrão de ICT, pois são muitos os fatores sociais que influenciam o índice. Entretanto, ao diagnosticar uma baixa CT, é importante promover ou restaurar a capacidade para o trabalho, pois há meios e instrumentos para tanto ${ }^{4,8}$.

Outro fator que influencia negativamente a capacidade para o trabalho é a realização de tare- 
fas com demandas de carga física. Esta constatação se deu em ao menos nove estudos $4,26,29,30,35,40-42,47$.

Ao investigar o ICT com relação aos trabalhos com demandas mentais, a literatura apresentou resultados variados. Cargos com demandas mentais predominantes estão associados a uma boa CT em oito casos $^{6,14,28,37,39,42,44,47}$. Em outros cinco casos $^{4,33,34,41,46}$, cargos com demandas mentais foram associados negativamente ao ICT. Em um dos casos, a demanda física e mental, mista, foi associada à chance de baixo $\mathrm{ICT}^{30}$. Ainda, quatro estudos apontam que os recursos mentais declinam com o passar da idade ${ }^{12,29,33,40}$.

Vislumbrou-se que as cargas física e mental no trabalho não apresentam um resultado uniforme quanto à influência sobre o ICT. Foi possível extrair do conteúdo dos artigos que há uma tendência relevante quando a demanda física está presente no cargo, mas este fator pode estar associado a outras variáveis, como posturas inadequadas ou estresse. Fato semelhante também ocorreu nos casos de cargos com demanda mental, onde houve maior variação de resultados, incluindo-se momentos em que a variável influencia positiva ou negativamente sobre $o$ ICT. Isto demonstrou que se faz necessário que o pesquisador esteja atento às peculiaridades de cada categoria profissional, e que os aspectos da saúde ocupacional vão além da definição de trabalho com demanda física ou mental.

Também houve constatação de que o gênero mulher está associado negativamente à CT. De acordo com os resultados, o fato das mulheres exercerem um papel fundamental na criação dos filhos e também na realização de trabalhos domésticos, impõe a elas uma jornada de trabalho dupla, às vezes até tripla, o que faz com que os ICT de mulheres estejam ligeiramente abaixo aos resultados obtidos por homens, conforme demonstrados em seis estudos ${ }^{16,28,30,34,35,48}$.

A responsabilidade sobre a renda familiar e a necessidade de trabalhar em dois empregos também são apontados como fatores que induzem à redução da CT. Estas variáveis estiveram associadas ao ICT em quatro artigos do portfólio ${ }^{28,34,43,48}$. Esta investigação ganha maior repercussão quando revelado que a responsabilidade sobre a renda familiar está correlacionada a baixos índices de ICT. A carga atribuída ao responsável pela subsistência da família impõe a este indivíduo condições de maior pressão e preocupação sobre o papel que ele desempenha como líder do núcleo familiar.

Outros 12 estudos relatam que a baixa capacidade para o trabalho também pode existir devido à presença de doenças ${ }^{15,38,44}$, em especial: estresse (quatro casos) ${ }^{18,31,41,46}$; a depressão, um estudo $^{27}$; problemas no sistema osteomuscular, em 2 situações ${ }^{16,29}$; e dificuldades com sono foram apontadas em 2 estudos ${ }^{35,48}$. Estes resultados corroboram para a utilização do ICT como uma ferramenta ergonômica e de gestão da saúde ocupacional, servindo de instrumento eficaz no acompanhamento da melhora da saúde do trabalhador com relação a sua capacidade laboral.

Alguns estudos também revelam variáveis que não demonstram significância estatística para o ICT, como a ingestão de bebidas alcoóli$\operatorname{cas}^{39}$ ou fumar ${ }^{47}$. Isto pode significar o chamado efeito "trabalhador saudável" 28 , sustentado na hipótese de que os trabalhadores com problemas de alcoolismo ou tabagismo não são contratados pelas empresas, estão afastados do trabalho na época da pesquisa, ou porque não aceitaram participar da pesquisa.

Enquanto os fatores que agem negativamente na saúde e capacidade do trabalhador devem ser observados, sobretudo no sentido de prevenir ou eliminar os riscos, outros fatores devem ser observados para promover a boa CT.

Programas e ações voltadas a promover a CT tem resultado positivo, mas devem ser tomados permanentemente, pois seus benefícios são alcançados em médio e longo prazos. Destacam-se entre as principais ações: melhorias ergonômicas, como eliminação de esforço repetitivo, melhorias nas posturas dos trabalhadores e postos de trabalho, relatados em quatro estudos ${ }^{6,26,33,34}$; e a prática regular de exercícios físicos, apontada por 11 artigos como variável que contribui para a melhoria da capacidade para o trabalho $^{15,18,26-28,30,32,39-41,47}$.

Outro aspecto relevante é o envolvimento do indivíduo com atividades de lazer e hobbies artísticos, relatado em três $\operatorname{casos}^{4,26,28}$. Ainda, a melhoria de situação econômica ${ }^{39}$, estabilidade no emprego $^{43}$, satisfação no trabalho ${ }^{27,33}$, a redução de carga horária ${ }^{18,41}$, foram associados aos trabalhadores com maiores ICT.

\section{Conclusões}

A presente pesquisa tratou de uma revisão sistemática da literatura com relação ao tema Capacidade para o Trabalho e Envelhecimento Funcional. Analisou-se o conteúdo de um portfólio de artigos de uma forma sistêmica, por meio da aplicação de lentes conceituais, destacando pontos fortes e fracos em cada lente. Este processo evidenciou a eficiência do PROKNOW-C para 
revisões de literatura, demonstrando rigor na obtenção dos resultados e, ainda, profundidade nas análises.

Uma limitação do estudo foi a utilização de apenas duas bases de dados. Em estudos futuros podem ser utilizadas bases de dados específicas da área da saúde.

Como principais resultados, destaca-se que o ICT é uma ferramenta amplamente difundida no campo da saúde ocupacional, e se mostrou eficiente na mensuração da capacidade para o trabalho. Esta capacidade, por sua vez, é um im- portante indicativo para o diagnóstico do envelhecimento funcional, sobretudo quando este processo ocorre de modo precoce.

As associações entre o ICT e dados sociodemográficos representaram uma parcela importante dos estudos, pois são fatores da vida social do indivíduo que podem influenciar em sua vida laboral. Destarte, a investigação de hábitos sociais contribui para avaliar se a capacidade para o trabalho é influenciada ou não por estes hábitos, e, por via de consequência, a existência de um envelhecimento funcional precoce.

\section{Colaboradores}

JE Linhares foi responsável pela concepção do estudo, redação geral do trabalho e realização da busca bibliográfica. SC Bortoluzzi atuou na utilização da ferramenta $P R O K N O W-C$ e na análise crítica. SLR Pessa e RP Luz atuaram na análise crítica e redação final do texto. 


\section{Referências}

1. Organização Mundial da Saúde (OMS). Envelhecimento e Saúde. OMS. setembro 2015. [acessado 2016 Jan 16]. Disponível em: http://www.who.int/mediacentre/ factsheets/fs404/en/\#

2. Martinez MC, Latorre MDRDDO. Health and work ability of workers of the electricity sector in Sao Paulo. Cien Saude Colet 2008; 13(3):1061-1073.

3. Instituto Brasileiro de Geografia e Estatística (IBGE). Projeção da População do Brasil por sexo e idade: 20002060. 2013. [acessado 2015 Nov 20]. Disponível em: http://www.ibge.gov.br/home/estatistica/populacao/ projecao_da_populacao/2013/default.shtm

4. Tuomi K, Huuhtanen P, Nykyri E, Ilmarinen J. Promotion of work ability, the quality of work and retirement. Occup Med 2001; 51(5):318-324.

5. Fischer FM, Borges NDS, Rotenberg L, Latorre MRDOL, Soares NS, Rosa PLFSR, Nagai R, Landsbergis P. A (in)capacidade para o trabalho em trabalhadores de enfermagem. Rev. Bras. Med. Trab. 2005; 3(2):97-103.

6. Martinez MC, Latorre MRO. Health and work ability among office workers. Rev Saude Publica 2006; 40(5):851-858.

7. Amorim JSCD, Salla S, Trelha CS. Factors associated with work ability in the elderly: systematic review. Rev Bras Epidemiol 2014; 17(4):830-841.

8. Tuomi K, Ilmarinen J, Jahkola A, Katajarinne L, Tulkki A. Indice de capacidade para o trabalho. São Carlos: EDUFSCAR; 2005.

9. Tuomi K, IImarinen J, Eskelinen L, Jarvinen E, Toikkanen J, Klockars M. Prevalence and incidence rates of diseases and work ability in different work categories of municipal occupations. Scand J Work Environ Health 1991; 17(Supl. 1):67-74.

10. Ilmarinen J, Tuomi K, Eskelinen L, Nygard CH, Huuhtanen P, Klockars M. Background and objectives of the Finnish research project on aging workers in municipal occupations. Scand J Work Environ Health 1991; 17(Supl. 1):7-11.

11. Tuomi K, Ilmarinen J, Klockars M, Nygard CH, Seitsamo J, Huuhtanen P, Martikainen R, Aalto L. Finnish research project on aging workers in 1981-1992. Scand J Work Environ Health 1997; 23(Supl. 1):7-11.

12. Ilmarinen J. Work ability-a comprehensive concept for occupational health research and prevention. Scand J Work Environ Health 2009; 35(1):1-5.

13. Costa CSND, Freitas EGD, Mendonça LCDS, Alem MER, Coury HJCG. Work ability and quality of life of brazilian industrial workers. Cien Saude Colet 2012; 17(6):1635-1642.

14. Martinez M, Latorre MRDO, Fischer FM. Validity and reliability of the Brazilian version of the Work Ability Index questionnaire. Rev Saude Publica 2009; 43(3):525-532.

15. Padula RS, Comper MLC, Moraes SA, Sabbagh C, Pagliato Junior W, Perracini MR. The work ability index and functional capacity among older workers. Braz J Phys Ther 2013; 17(4):382-391.

16. Paula ÍR, Marcacine PR, Castro SS, Walsh IAP. Capacidade para o trabalho, sintomas osteomusculares e qualidade de vida entre agentes comunitários de saúde em Uberaba, Minas Gerais. Saude Soc 2015; 24(1):152-164.
17. Sampaio RF, Coelho CM, Barbosa FB, Mancini MC Parreira VF. Work ability and stress in a bus transportation company in Belo Horizonte, Brazil. Cien Saude Colet 2009; 14(1):287-296.

18. Karazman R, Kloimüller I, Geissler H, Karazman-Morawetz I. Effects of ergonomic and health training on work interest, work ability and health in elderly public urban transport drivers. International Journal of Industrial Ergonomics 2000; 25(5):503-511.

19. Ensslin L, Ensslin SR, Lacerda RTO, Tasca JE. ProKnow-C, Knowledge Development Process - Constructivist. Processo técnico com patente de registro pendente junto ao INPI. Rio de Janeiro: INPI; 2010.

20. Lacerda RTDO, Ensslin L, Ensslin SR. Uma análise bibliométrica da literatura sobre estratégia e avaliação de desempenho. Gestão \& Produção 2012; 19(1):59-78.

21. Lacerda RTDO, Ensslin L, Ensslin SR. Research opportunities in strategic management field: a performance measurement approach. Int $J$ Business Performance Management 2014; 15(2):158-174.

22. Tasca JE, Ensslin L, Ensslin SR, Alves MBM. An approach for selecting a theoretical framework for the evaluation of training programs. Journal of European Industrial Training 2010; 34(7):631-655.

23. Viegas CV, Bond AJ, Vaz CR, Borchardt M, Pereira GM, Selig PM, Varvakis G. Critical attributes of Sustainability in Higher Education: A categorisation from literature review. Journal of Cleaner Production 2016; 126:260-276.

24. Guerrero-Botea VP, Moya-Anegón F. A further step forward in measuring journals' scientific prestige: The SJR2 indicator. Journal of Informetrics 2012; 6(4):674688.

25. Coordenação de Aperfeiçoamento de Pessoal de Nível Superior (CAPES). Portal de Periódicos CAPES/ MEC - Estatísticas de Uso. [acessado 2016 Jan 30]. Disponível em: http://www.periodicos.capes.gov.br/index. php? $\mathrm{mn}=0 \& \mathrm{smn}=0$

26. Chan G, Tan V, Koh D. Ageing and fitness to work. Occup Med 2000; 50(7):483-491.

27. Kumashiro M. An approach of ergonomics and management in occupational health for a society of aging workers. Journal of Japan Industrial Management Association 2014; 65(2):124-130.

28. Monteiro MI, Fernandes ACP. Capacidade para o trabalho de trabalhadores de empresa de tecnologia da informação. Rev Bras Enferm 2006; 59(5):603-608.

29. Huuhtanen P, Nygård $\mathrm{CH}$, Tuomi K, Martikainen R. Changes in stress symptoms and their relationship to changes at work in 1981-1992 among elderly workers in municipal occupations. Scand J Work Environ Health 1997; 1(Supl. 1):36-48.

30. Ilmarinen J, Tuomi K, Klockars M. Changes in the work ability of active employees over an 11-year period. Scand J Work Environ Health 1997; 23(Supl. 1):49-57.

31. Saltychev M, Laimi K, Oksanen T, Pentti J, Kivimäki M, Vahtera J. Does perceived work ability improve after a multidisciplinary preventive program in a population with no severe medical problems? The Finnish Public Sector Study. Scand J Work Environ Health 2013; 39(1):57-65. 
32. Nurminen E, Malmivaara A, Ilmarinen J, Ylöstalo P, Mutanen P, Ahonen G, Aro T. Effectiveness of a worksite exercise program with respect to perceived work ability and sick leaves among women with physical work. Scand J Work Environ Health 2002; 28(2):85-93.

33. Chiu MC, Wang MJJ, Lu CW, Pan SM, Kumashiro M, Ilmarinen J. Evaluating work ability and quality of life for clinical nurses in Taiwan. Nurs Outlook 2007; 55(6):318-326.

34. Vasconcelos SP, Fischer FM, Reis AOA, Moreno CRC. Factors associated with work ability and perception of fatigue among nursing personnel from Amazonia. Rev Bras Epidemiol 2011; 14(4):688-697.

35. Vedovato TG, Monteiro I. Health Conditions and Factors Related to the Work Ability of Teachers. Ind Health 2014; 52(2):121-128.

36. Larsson A, Karlqvist L, Westerberg M, Gard G. Identifying work ability promoting factors for home care aides and assistant nurses. BMC Musculoskelet Disord 2012; 13:1.

37. Williams SN, Crumpton LL. Investigating the work ability of older employees. International Journal of Industrial Ergonomics 1997; 20(3):241-249.

38. Pranjic N, Males-Bilic L, Beganlic A, Mustajbegovic J. Mobbing, stress, and work ability index among physicians in Bosnia and Herzegovina: Survey study. Croat Med J 2006; 47(5):750-758.

39. Tuomi K, Vanhala S, Nykyri E, Janhonen M. Organizational practices, work demands and the well-being of employees: a follow-up study in the metal industry and retail trade. Occup Med 2004; 54(2):115-121.

40. Ilmarinen J. Physical requirements associated with the work of aging workers in the European Union. Exp Aging Res 2002; 28(1):7-23.

41. Klasan A, Madzarac G, Milosevic M, Mustajbegovic J, Keleuva S. Predictors of lower work ability among emergency medicine employees: the Croatian experience. Emerg Med J 2013; 30(4):275-279.

42. Seibt R, Spitzer S, Blank M, Scheuch K. Predictors of work ability in occupations with psychological stress. $J$ Public Health 2008; 17(1):9-18.

43. Marqueze EC, Moreno CRDC. Job satisfaction and work ability among college educators. Psicol Estud 2009; 14(1):75-82.
44. Giannini SPP, Latorre MDRDDO, Fischer FM, Ghirardi ACDAM, Ferreira LP. Teachers' voice disorders and loss of work ability: a case-control study. J Voice 2015; 29(2):209-217.

45. De Zwart BCH. Test-retest reliability of the Work Ability Index questionnaire. Occup Med 2002; 52(4):177181.

46. Kloimüller I, Karazman R, Geissler H, Karazman-Morawetz I, Haupt H. The relation of age, work ability index and stress-inducing factors among bus drivers. International Journal of Industrial Ergonomics 2000; 25(5):497-502.

47. El Fassi M, Bocquet V, Majery N, Lair ML, Couffignal S, Mairiaux P. Work ability assessment in a worker population: comparison and determinants of Work Ability Index and Work Ability score. BMC Public Health 2013; 13:305.

48. Fischer FM, Borges FNDS, Rotenberg L, Latorre MDRDDO, Soares NS, Rosa PLFS, Teixeira LR, Nagai R, Steluti J, Landsbergis P. Work ability of health care shift workers: What matters? Chronobiol Int 2006; 23(6):1165-1179.

49. Creswell JW. Projeto de Pesquisa: método qualitativo, quantitativo e misto. $3^{\text {a }}$ ed. Porto Alegre: Artemed; 2010.

50. Marafon AD, Ensslin L, Lacerda RT, Ensslin SR. Avaliação de desempenho na gestão de $\mathrm{P} \& \mathrm{D}$ - revisão sistêmica literária. P\&D em Engenharia de Produção 2012; 10(2):171-194.

51. Lizot M, Júnior PPDA, Magacho CS, Bortoluzzi SC. Avaliação de Desempenho na Gestão da Produção: Análise Bibliométrica e Sistêmica da Literatura Internacional. In: $9^{\circ}$ Congresso IBEROAMERICANO de Contabilidade e Gestón, 2015; Florianópolis, SC.

52. Miguel PAC. Metodologia de pesquisa para engenharia de produção e gestão de operações. Rio de Janeiro: Elsevier, ABEPRO; 2012.

Artigo apresentado em 08/11/2016

Aprovado em 06/02/2017

Versão final apresentada em 08/02/2017 